\title{
Long-term therapy with intravenous zoledronate increases the number of nonattached osteoclasts
}

\author{
Maria José H. Nagata ${ }^{\mathrm{a}}$, , Michel R. Messora ${ }^{\mathrm{b}}$, Cristina Antoniali ${ }^{\mathrm{c}}$, Stephen E. Fucini a, d, \\ Natália de Campos ${ }^{a}$, Natália M. Pola ${ }^{e}$, Carolina S. Santinoni ${ }^{f}$, Flávia A.C. Furlaneto ${ }^{\text {b }}$, \\ Edilson Ervolino ${ }^{\mathrm{c}}$ \\ a University Estadual Paulista - UNESP, Dental School of Araçatuba, Department of Surgery and Integrated Clinic, Araçatuba, SP, Brazil \\ ${ }^{\mathrm{b}}$ University of São Paulo - USP, Ribeirão Preto School of Dentistry, Department of Oral and Maxillofacial Surgery and Traumatology and Periodontology, \\ Ribeirão Preto, SP, Brazil \\ ${ }^{\mathrm{c}}$ University Estadual Paulista - UNESP, Dental School of Araçatuba, Department of Basic Sciences, Araçatuba, SP Brazil \\ ' Private Practice, $\mathrm{NH}$, Hanover, USA \\ e Federal University of Pelotas - UFPel, Dental School of Pelotas, Department of Semiology and Clinic, Pelotas, RS, Brazil \\ ${ }^{\mathrm{f}}$ University Do Oeste Paulista - UNOESTE, Dental School of Presidente Prudente, Division of Periodontics, Presidente Prudente, SP, Brazil
}

\section{A R T I C L E I N F O}

\section{Article history:}

Paper received 14 October 2016

Accepted 10 August 2017

Available online 19 August 2017

\section{Keywords:}

Zoledronic acid

Tooth extraction

Bone regeneration

\begin{abstract}
A B S T R A C T
The purpose of this study was to investigate the influence of long-term therapy with intravenous zoledronate (ZA) on the healing of extraction sockets in rats. Forty rats, divided into groups $\mathrm{C}$ (Control) and $Z$ (Zoledronate), received intravenous injections of either saline solution or ZA for 24 weeks. Their right maxillary incisor was extracted. Euthanasia was performed at 7 or 28 days postoperative. Histomorphometric (Newly Formed Bone Area) and immunohistochemical (RANKL, OPG and TRAP) analyses were performed. Data were statistically analyzed (ANOVA, Tukey's test and Kruskal-Wallis, Dunn's Multiple Comparison test).Groups $C$ and $Z$ showed similar new bone area, RANKL and OPG immunolabeling. The number of TRAP-positive multinucleated cells was significantly higher in Group $Z$ than in Group C at 28 days. A significantly higher proportion of nonattached osteoclasts were seen in Group Z than in Group $C$ at both periods of analysis. Long-term therapy with intravenous ZA stimulated nonattached osteoclast formation in extraction sockets in rats, thus decreasing local bone resorption. However, it did not influence bone formation by osteoblasts.
\end{abstract}

๑) 2017 European Association for Cranio-Maxillo-Facial Surgery. Published by Elsevier Ltd. All rights reserved.

\section{Introduction}

Bisphosphonates (BPs) are antiresorptive drugs that are being used clinically to treat bone-related diseases, such as osteoporosis or cancer with bone metastasis (Williams et al., 2014). Users of these drugs are known to be at higher risk of developing osteonecrosis of the jaw (ONJ), which was first described by Marx (2003).

A systematic review and international consensus was published by a 'task force' sponsored by fourteen societies, including ASBMR, AAOMS, Canadian Association of Oral and Maxillofacial Surgeons, European Calcified Tissue Society, and Japanese Society for Bone

\footnotetext{
* Corresponding author. Rua José Bonifácio, 1193, CEP: 16015-050 Araçatuba, SP, Brazil.
}

E-mail address: mjnagata@foa.unesp.br (M.J.H. Nagata). and Mineral Research, among others. This task force defines ONJ as: 1) exposed bone in the maxillofacial region that does not heal within 8 weeks after identification by a health care provider; 2) exposure to an antiresorptive agent; and 3) no history of radiation therapy to the craniofacial region (Khan et al., 2015).

The incidence of $\mathrm{ONJ}$ is highest in the oncology patient population (1-15\%), where high doses of BPs are used at frequent intervals (Khan et al., 2015). In the osteoporosis patient population, ONJ development still remains controversial (Williams et al., 2014). The vast majority of case series have described ONJ occurring at sites that have had oral surgery, particularly at extraction sites (Barasch et al., 2011; Ferlito et al., 2011).

The pathophysiology of $\mathrm{ONJ}$ is not well understood. The development of ONJ appears to be multifactorial and related to the dose and duration of exposure to the antiresorptive agent, pre-existing 
oral disease profile, intervention with dentoalveolar oral surgery, and genetic polymorphisms (Khan et al., 2015).

Osteoclasts, which are thought to be the key cells involved in the effects of BPs on ONJ, have two distinct functions: bone resorption and maintenance of bone remodeling balance (Kim et al., 2016). Osteoprotegerin (OPG), receptor activator of nuclear factor- $\kappa \beta$ (RANK), and receptor activator of nuclear factor- $\kappa \beta$ ligand (RANKL) proteins are mediators of several cell processes, including bone metabolism. In bone metabolism, osteoblasts modulate osteoclast formation and bone resorption by producing OPG and RANKL (Manrique et al., 2015). OPG (Shalhoub et al., 1999; Suda et al., 1999; Manrique et al., 2015) is able to bind to RANKL, preventing it from binding to RANK. By binding to RANKL, OPG inhibits osteoclast maturation, thus inhibiting osteoclastogenesis as well as lymphocyte development. Any imbalance in the communication between OPG, RANK, and RANKL proteins can lead to alterations in the quality of the formed mineralized tissue (Shalhoub et al., 1999; Suda et al., 1999; Manrique et al., 2015). Some studies have demonstrated that BPs inhibit the expression of RANKL, increase levels of OPG, and reduce osteoclast differentiation (Nakagawa et al., 2015; Mackie et al., 2001; Viereck et al., 2002). In contrast, Cardemil et al. (2015) observed similar expression of RANKL and OPG in alveolar bone samples from patients with and without ONJ after BP treatment.

Studies (Bi et al., 2010; Hokugo et al., 2010; Aghaloo et al., 2011; Aguirre et al., 2012; Williams et al., 2014) have unequivocally shown osteoclasts (Baron et al., 2011; Williams et al., 2014) to be the key mediators in causing ONJ. Nonetheless, direct evidence of involvement and the extent to which osteoclasts contribute to ONJ pathogenesis remains elusive. It remains unclear whether ONJ development by BPs is associated with their inhibitory effects on bone-resorptive functions of osteoclasts or with the aberrant behavior of osteoclasts (e.g. secretion of cytokines to the surrounding environment) after BP uptake (Williams et al., 2014). Current literature remains inconclusive with respect to the presence, numbers, and location of mature osteoclasts (Reid and Cornish, 2011; Williams et al., 2014). Cytotoxic effects of BPs on cells other than osteoclasts (e.g. endothelial cells or oral mucosa cells) have further complicated the roles of osteoclasts in the pathophysiological characteristics of ONJ (Williams et al., 2014).

Several mechanisms have been proposed in an attempt to explain the pathophysiology of BP-related ONJ (BRONJ) (DinizFreitas et al., 2012; Silva et al., 2015), but we are far from understanding its pathogenesis. The pathophysiology of ONJ needs to be more clearly delineated using well-characterized animal models that lend themselves to better understanding the human condition (Khan et al., 2015). The purpose of this study was to histomorphometrically and immunohistochemically analyze the influence of long-term therapy with IV zoledronate (ZA) on the healing of healthy extraction sockets in rats.

\section{Material and methods}

\subsection{Animals and study design}

The experimental protocol was approved by the Univ. Estadual Paulista - UNESP, Dental School of Araçatuba Institutional Animal Care and Use Committee (\# 2007-001204), SP, Brazil, and is in accordance with the EU Directive 2010/63/EU. Forty healthy male rats (Rattus norvegicus albinus, Wistar), 3-4 months old, weighing 350-400 g (UNESP, Dental School of Araçatuba, Animal Care Unit) were randomly divided into two groups: $Z$ (zoledronate) and $C$ (control). Group Z received IV injections of ZA (Zometa ${ }^{\circledR} 4 \mathrm{mg}$, Novartis Pharmaceuticals Corporation, East Hanover, NJ, USA; $76 \mu \mathrm{g} / \mathrm{kg}$ of body weight diluted in sterile $0.9 \%$ sodium chloride) once every 4 weeks over the course of 24 weeks. This dose was calculated by the pharmacology researcher (CA) to be equivalent to that used for oncology treatments. Under identical conditions, group $C$ received IV injections of saline solution $(0.15 \mathrm{M})$ containing no ZA. The solutions were administered in the jugular vein.

Under general anesthesia, tooth (healthy) extractions were performed $24 \mathrm{~h}$ after the last IV injection of either solution. Each group was divided into two sub-groups for euthanasia at 7 or 28 days postoperative $(n=10)$. All animals were euthanized with an overdose of pentobarbital (Thiopentax, Cristália Produtos Químicos e Farmacêuticos Ltda., São Paulo, Brazil).

\subsection{Tooth extractions}

Following the six monthly injections ( $24 \mathrm{~h}$ after the last injection of either saline or ZA), the rats were anesthetized by an intramuscular injection of xylazine $(6 \mathrm{mg} / \mathrm{kg}$ body weight) and ketamine (70 $\mathrm{mg} / \mathrm{kg}$ body weight) and the healthy right maxillary incisor was extracted. The dental sockets were sutured.

\subsection{Tissue processing}

Animals were euthanized at either 7 or 28 days postoperative. The area of the extraction socket and the surrounding tissues were removed en bloc. The blocks obtained were fixed in 10\% neutral formalin, rinsed with water, and then decalcified in $10 \%$ ethylenediaminetetraacetic acid (EDTA) solution. Following decalcification, they were processed and embedded in paraffin. Serial sections $6 \mu \mathrm{m}$ thick were cut in a longitudinal direction and stained either with hematoxylin and eosin $(\mathrm{H} \& \mathrm{E})$ for histomorphometric analyses, or subjected to the indirect immunoperoxidase detection method.

For the immunohistochemical reactions, the histological sections were deparaffinized and rehydrated through a graded series of ethanol. For antigen retrieval, the slides were incubated in a buffer solution (Diva Decloaker ${ }^{\mathbb{B}}$, Biocare Medical, CA, USA) in a pressurized chamber (Decloaking ChamberTM, Biocare Medical, CA, USA) at $95{ }^{\circ} \mathrm{C}$ for $10 \mathrm{~min}$. At the end of each step of the immunohistochemical reaction, the histological slides were washed with phosphate buffer solution $(0.1 \mathrm{M}, \mathrm{pH} 7.4)$. The slides were immersed in 3\% hydrogen peroxide for $1 \mathrm{~h}$ and $1 \%$ albumin bovine serum for $12 \mathrm{~h}$ to block the endogenous peroxidase and nonspecific sites, respectively. The slides containing samples of each of the experimental groups were divided into three batches and each batch was incubated with one of the following primary antibodies: goat anti-rat RANKL (1:100; Santa Cruz Biotechnology, Santa Cruz, CA, USA), rabbit anti-rat OPG (1:100; Santa Cruz Biotechnology, Santa Cruz, CA, USA) or goat anti-rat TRAP (1:180; Santa Cruz Biotechnology, Santa Cruz, CA, USA). The sections were incubated with a biotinylated secondary antibody for $2 \mathrm{~h}$ and subsequently treated with a streptavidin-horseradish peroxidase conjugate for $1 \mathrm{~h}$ (Universal Dako Labeled HRP Streptavidin-Biotin Kit ${ }^{\mathbb{R}}$, Dako Laboratories, CA, USA). The reaction was developed using the chromogen 3,3'-diaminobenzidine (DAB chromogen $\mathrm{Kit}^{\circledR}$, Dako Laboratories, CA, USA) and counterstained with Harris hematoxylin. All samples were accompanied with a negative control.

\subsection{Image analysis procedure}

Five histological sections, representing the center of the original extraction socket, were selected for histological/histometric (2 sections) and immunohistochemical (3 sections) analyses. They were performed by examiners, calibrated and masked with respect to the treatment rendered, using image-analysis software (Axiovision 4.8.2, Carl Zeiss MicroImaging GmbH, Jena, Germany). 
The histological analysis was performed by a certified histologist (EE), assessing the following parameters in the extraction socket and surrounding tissues: 1) nature and degree of inflammation; 2) extension of the inflammatory process to the surrounding tissues; 3) presence and extension of tissue necrosis; 4) vascular status; 5) pattern of the extracellular matrix structure of the preexisting and newly formed tissues; 6) cellularity pattern of the preexisting and newly formed tissues.

The histometric analysis was performed by another examiner (NMP). The images of the histological sections were saved on a computer after capture by a digital camera connected to a light microscope (Axiostar Plus, Carl Zeiss MicroImaging GmbH, Gottingen, Germany) with an original magnification of $\times 200$. The following criteria were used to standardize the histometric analysis of the digital images: a) delineation of the total area (TA) to be analyzed, which corresponded to the entire area of the original extraction socket; b) the newly formed bone area (NFBA), which was delineated within the confines of the TA and calculated as a percentage of TA.

The immunohistochemical analysis was performed by a certified histologist (EE). Criteria based on the work of Nagata et al. (2009) were used to standardize the TRAP immunohistochemical analysis of the digital image (histological section captured with an original magnification of $\times 400$ ). The TA corresponded to the entire area of the original extraction socket. TRAP-positive mononuclear cells and TRAP-positive multinucleated cells were quantified within the confines of the TA. In addition, the number of nonattached TRAP-positive multinucleated cells was quantified and their proportion in relation to the total number of TRAP-positive multinucleated cells calculated.

The following criteria were used to standardize the RANKL and OPG immunohistochemical analyses of the digital image (histological sections captured with an original magnification of $\times 400$ ):

a) Measurements of both the buccal and palatal walls (BW and PW) of the socket were carried out; each measurement (BW and PW) was then divided into three equal parts using a series of four equidistant marks. The most coronal BW mark was connected to the most coronal PW mark via a straight line, proceeding apically in a sequential manner until the most apical BW mark was connected to the most apical PW mark via a straight line as well. The area between two of the straight lines corresponding to the middle third of the extraction socket was identified.

b) RANKL-positive and OPG-positive cells were then semiquantified within the confines of the middle third of the socket area. The immunostaining score was based on Theodoro et al. (2016) according to the following criteria: 0 - absence of immunolabeling; 1 - extremely low pattern of immunolabeling; 2 - low pattern of immunolabeling; 3 - moderate pattern of immunolabeling; 4 - high pattern of immunolabeling; 5 extremely high pattern of immunolabeling.

\subsection{Statistical analysis}

The data normality and homogeneity of variances were verified. Each parameter was evaluated separately. For NFBA, number of TRAP-positive mononuclear cells, number of TRAP-positive multinucleated cells, and the proportion of nonattached TRAP-positive multinucleated cells, the significance of differences between groups was determined by an analysis of variance (ANOVA), followed by a post hoc Tukey's test when the ANOVA suggested a significant difference between groups $(p<0.05)$. For RANKL and OPG scores, the significance of differences between groups was determined by a Kruskal-Wallis test, followed by a post hoc Dunn's multiple comparison test when the Kruskal-Wallis test suggested a significant difference between groups $(p<0.05)$ (GraphPad Prism version 5.0 for Windows, San Diego, CA, USA).

\section{Results}

\subsection{Histomorphometric analyses}

At 7 days postoperative, the cervical third of the extraction socket in both groups was occupied by remnants of blood clot and connective tissue with a network of collagen fibers, and a large number of fibroblasts and blood vessels. A thin network of immature bone trabecule, which were surrounded by osteoblasts with morphologic characteristics of intense bone matrix synthesis, was observed in the middle (Fig. 1A and B) and apical thirds of the socket. Sparsely distributed osteoclasts were seen associated with resorption lacunae on the surface of some bone trabecule. Many giant, hypernucleated, nonattached osteoclasts were observed in group $Z$.

At 28 days postoperative, the extraction socket in both groups was filled with newly formed bone. In the cervical third of the socket, wide marrow spaces with highly vascularized loose connective tissue were surrounded by thin bone trabecule. Thick bone trabecule were observed in the middle (Fig. 1C and D) and apical thirds of the socket. Some bone trabecule were surrounded by active osteoblasts, while others were surrounded by bone lining cells. An increased number of osteoclasts was observed compared with the 7 day specimens, many of which were giant, hypernucleated, nonattached osteoclasts (Fig. 2E). The number of osteoclasts was greater in group $\mathrm{Z}$ than in group C. Most osteoclasts were active in group $\mathrm{C}$, while in group $\mathrm{Z}$ most of them were nonattached.

The means and standard deviations of NFBA (in \%) for each group at both 7 and 28 days postoperative, as well as the results of the intra- and intergroup comparisons, are presented in Fig. 1E.

\subsection{Immunohistochemical analysis}

The immunohistochemical technique used for detecting RANKL, OPG, and TRAP yielded high specificity in the detection of these proteins, as evidenced by the total absence of staining in the negative control for the immunohistochemical reactions. The immunolabeled cells had a brownish color that was confined to the cytosolic compartment.

TRAP immunolabeling was observed in osteoclast precursor cells (Fig. 2A), as well as in mature attached (Fig. 2C) and nonattached osteoclasts (Fig. 2E). RANKL and OPG immunolabeling was predominantly expressed in osteoblasts and in a few fibroblasts of the connective tissue adjacent to the newly formed bone trabecule. RANKL and OPG immunolabeling patterns at 28 days postoperative in both groups are presented in Fig. 3A-D.

The means and standard deviations of TRAP-positive mononuclear and multinucleated cells, and the proportion of nonattached osteoclasts for each group at both 7 and 28 days postoperative, as well as the results of the intra- and intergroup comparisons, are presented in Fig. 2B, D and F.

The medians and interquartile deviations of the scores attributed to the expressions of RANKL and OPG for each group at both 7 and 28 days postoperative, as well as the results of the intra- and intergroup comparisons, are presented in Fig. $3 \mathrm{E}$ and $\mathrm{F}$.

\section{Discussion}

The aim of this study was to histomorphometrically and immunohistochemically analyze the influence of long-term 


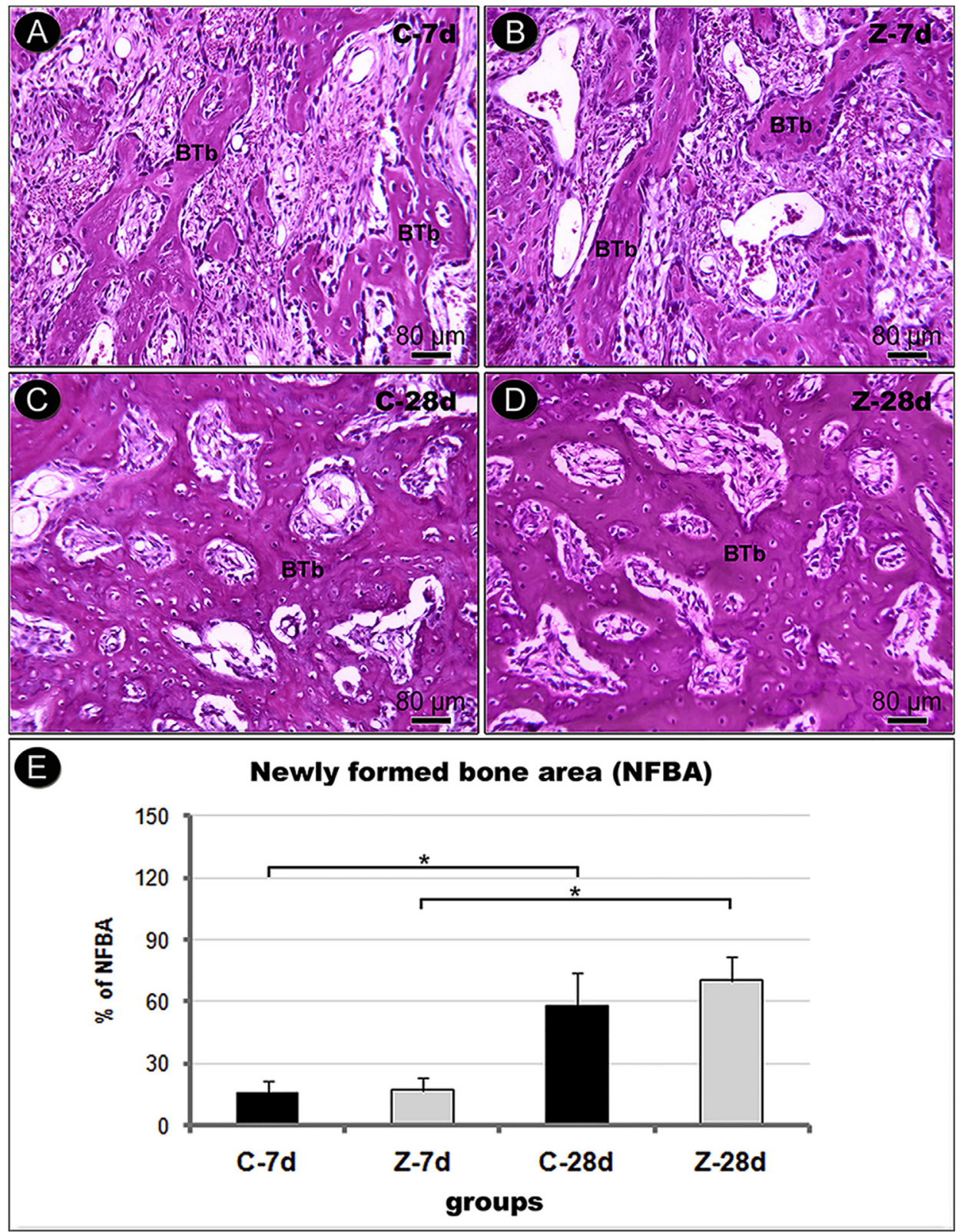

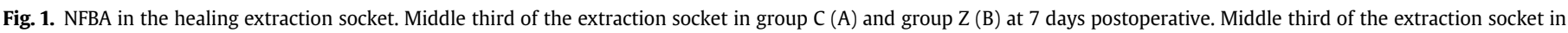

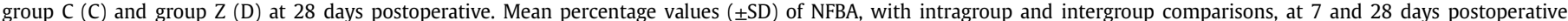
$\left({ }^{*} p<0.05\right)(\mathrm{E})$. Hematoxylin and eosin bars $=80 \mu \mathrm{m}$. NFBA $=$ newly formed bone area. $\mathrm{BTb}=$ bone trabecule.

therapy with IV ZA on the healing of healthy extraction sockets in rats. To date, no direct causal link has been established between bisphosphonate (BF) treatment and the occurrence of osteonecrosis of the jaw (ONJ) (Khan et al., 2015; Howie et al., 2015). However, it is now widely accepted that long-term intravenous BF therapy carries a much higher risk of ONJ, and that such risk is highest after dental extraction (Reid and Cornish, 2011; Howie et al., 2015).

Although multiple studies have reported the occurrence of BPrelated ONJ-like (BRONJ) features in animal models, success rates and reproducibility have been highly variable (Kobayashi et al., 2010; Ali-Erdem et al., 2011; Aguirre et al., 2012; Kuroshima and Yamashita, 2013; Howie et al., 2015). One explanation for this variability of results may be the use of experimental models involving the extraction of healthy teeth. Recently, Soundia et al. (2016) showed interesting findings that supported the critical role of dental disease in the pathogenesis of ONJ, not only as the instigating cause for tooth extraction, but also as a compounding factor in ONJ development and pathophysiology. Our study corroborated those findings, once we observed that no clinical or histopathological signs of ONJ were observed in the animals in group $\mathrm{Z}$ after extraction of healthy teeth. The histomorphometric analysis revealed that the amount of newly formed bone was similar in groups $\mathrm{Z}$ and $\mathrm{C}$ for both periods of evaluation ( 7 and 28 days postoperative). Our results are also supported by other studies (Kuroshima and Yamashita, 2013; Kobayashi et al., 2010) that did not observe ONJ-like lesions in mice that had been treated with ZA alone and undergone healthy tooth extraction. However, Kobayashi et al. (2010) did find that ZA delayed wound healing of the extraction socket by inhibiting both osteogenesis and angiogenesis. The conflicting results might be attributable to great variations in 

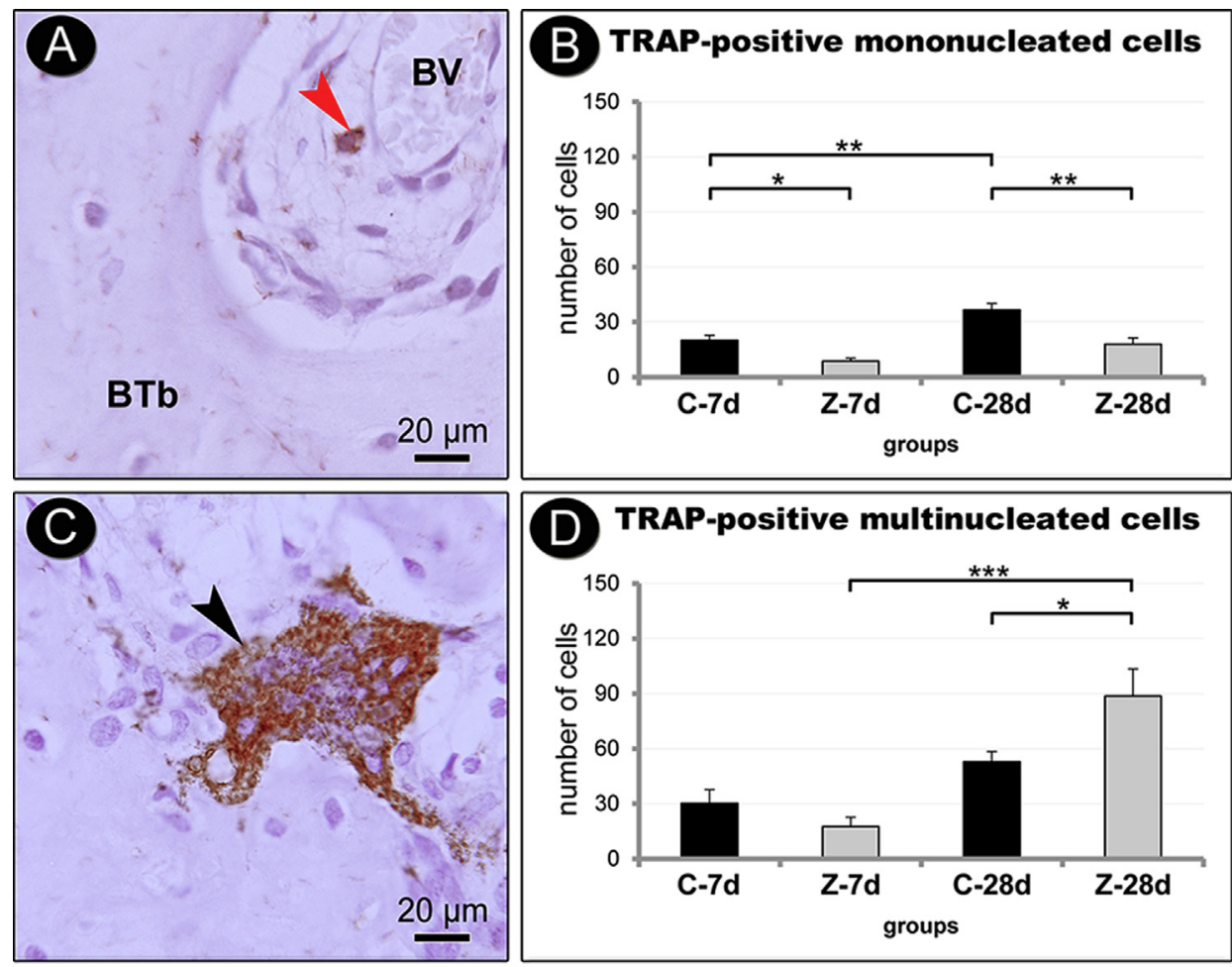

\section{TRAP-positive multinucleated cells}
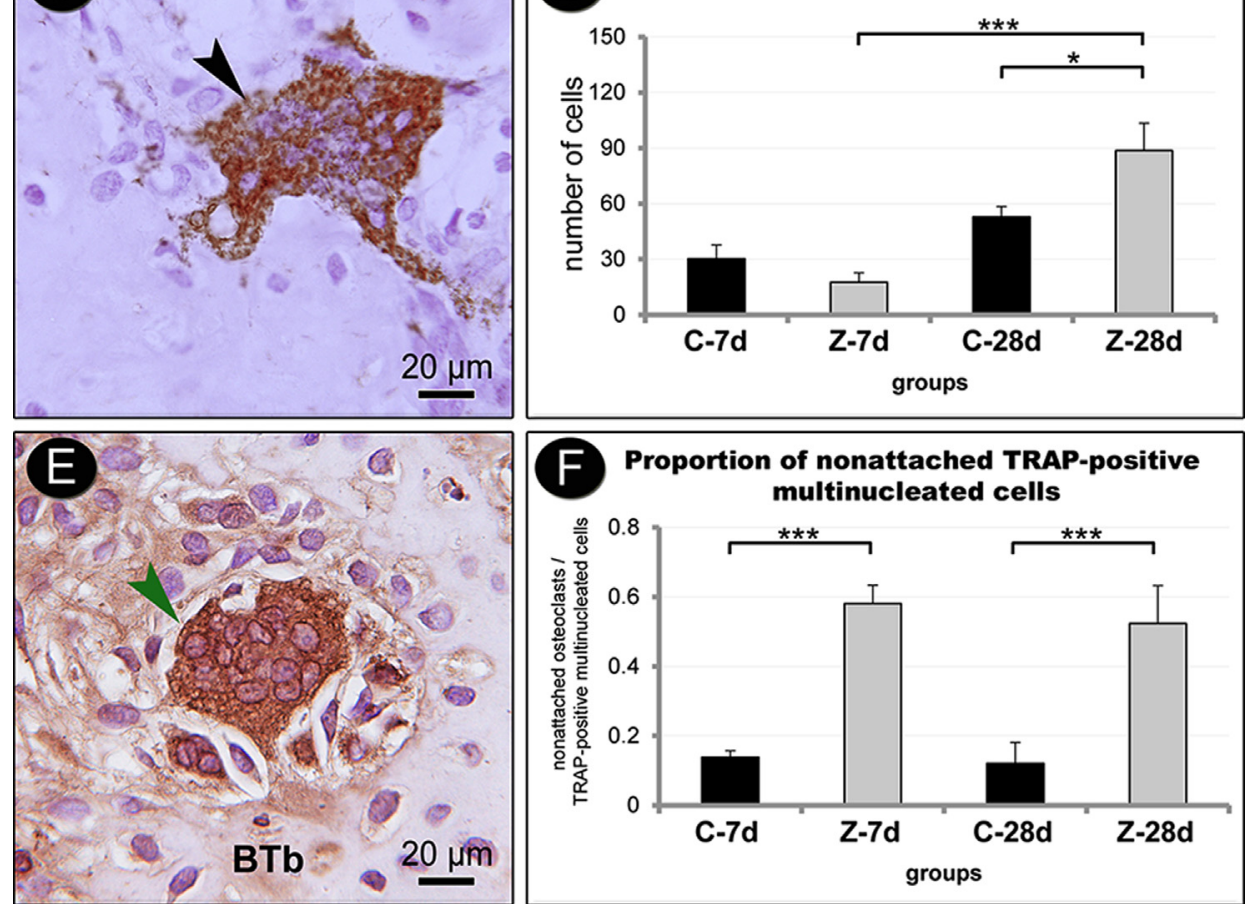

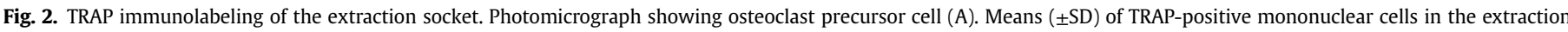

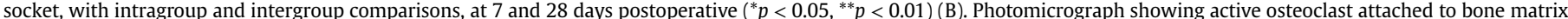

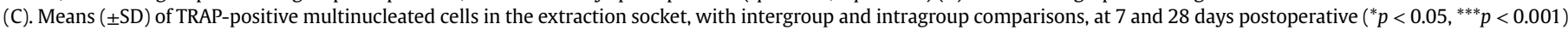

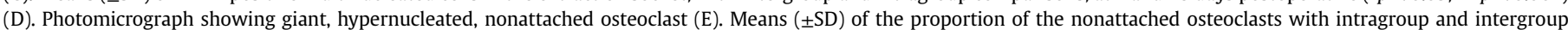

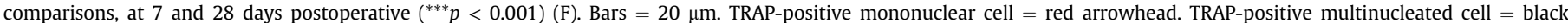
arrowhead. Giant, hypernucleated, nonattached osteoclast $=$ green arrowhead. BTb $=$ bone trabecule. $\mathrm{BV}=$ blood vessel.

the ZA protocols (dose, dosing interval, and duration of the therapy, both before and after tooth extraction) used in the studies.

The maximum duration of therapy with ZA in our study was 28 days. Several previous studies used this same period to investigate the effects of ZA after tooth extraction (Mada et al., 2017; Silva et al., 2015; Soundia et al., 2016; Marino et al., 2012; Ali-Erdem et al., 2011). Marino et al. (2012) observed that all rats treated with ZA presented bone necrosis at 4 weeks after tooth extraction. It is also important to highlight that some studies have demonstrated ONJ earlier than 4 weeks after tooth extraction in rats treated with BPs (Barba-Recreo et al., 2014; Williams et al., 2014).

Because the majority of the cases of ONJ have occurred with the use of high-dose IV BPs in the oncology patient population, the dose of ZA in the our study ( $76 \mu \mathrm{g} / \mathrm{kg}$ body weight) was calculated by the pharmacology researcher (CA) to simulate a human protocol for this drug and type of therapy. Similar doses were used in other studies (Hokugo et al., 2010; Aguirre et al., 2012) that have also been conducted to evaluate the influence of high-dose IV ZA, referred as an 'oncologic dose' by Aguirre et al. (2012).

In our study, ZA did not affect the RANKL/OPG ratio. The RANKL/ OPG ratio is an important determinant of bone mass in normal bone remodeling (Gong et al., 2017). Pan et al. (2004) examined the effect of ZA on the expression of RANKL/OPG in primary osteoblast-like cells derived from human bone and found that ZA did not significantly affect RANKL/OPG gene expression. Anti-RANKL agents such as denosumab prevented RANKL from binding to RANK and thereby inhibiting the development, activation, and differentiation of osteoclasts (Baron et al., 2011). According to Nakagawa et al. (2015) this is different from the mechanism of action of BPs, which bind to bone minerals and likely inhibit osteoclast differentiation mainly after being taken up by osteoclasts at sites of bone resorption.

In our study, the number of TRAP-positive multinucleated cells (osteoclasts) was significantly higher in group $\mathrm{Z}$ than in group $\mathrm{C}$ at 28 days postoperative. In addition, a significant increase in the 


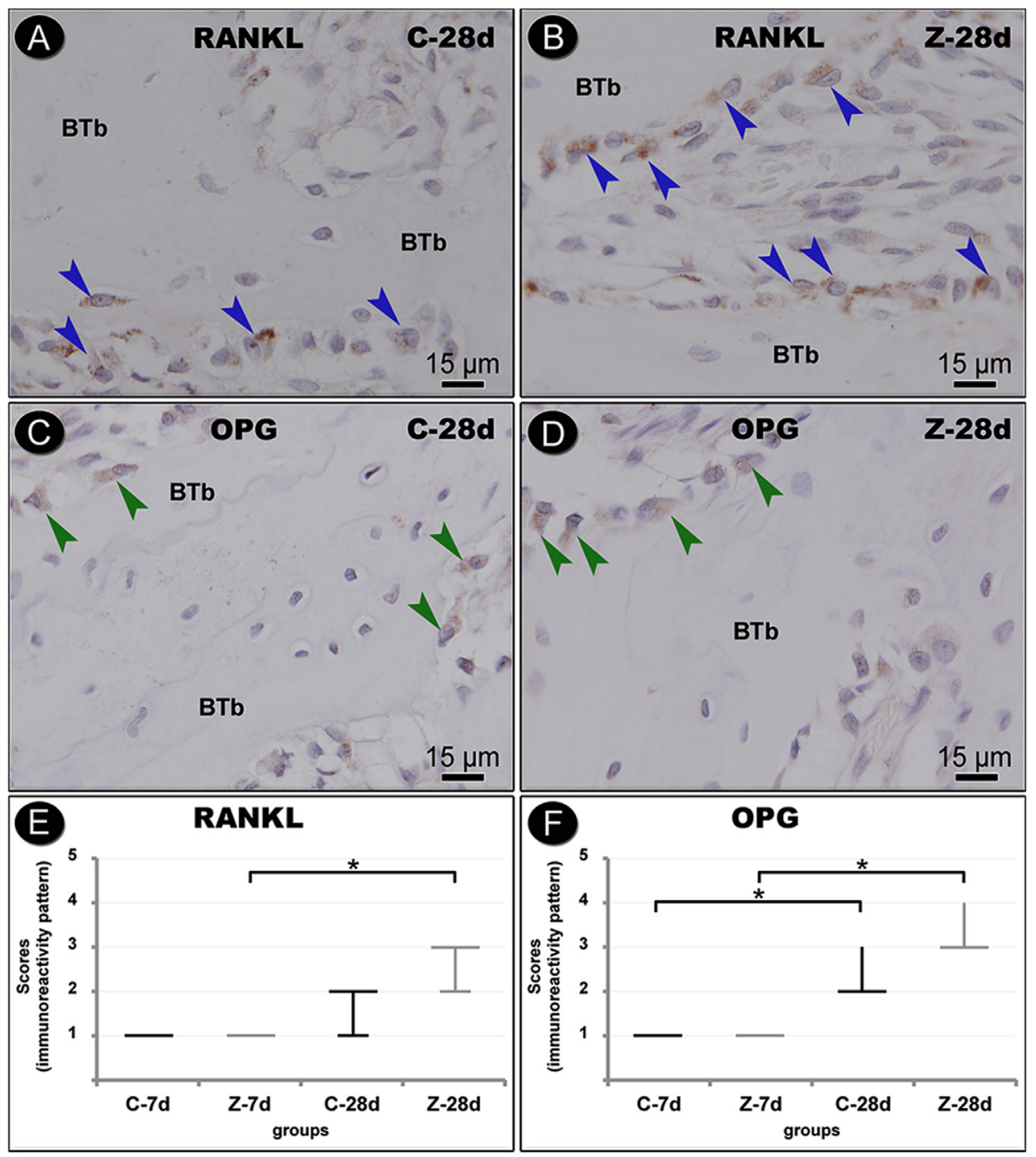

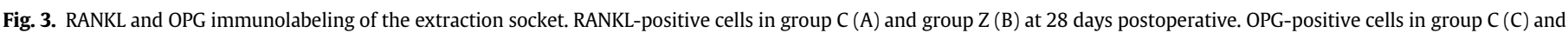

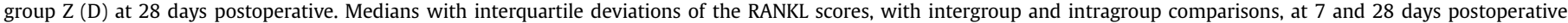

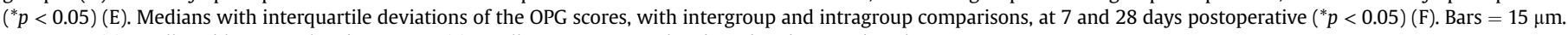
RANKL-positive cells $=$ blue arrowheads. OPG-positive cells $=$ green arrowheads. $\mathrm{BTb}=$ bone trabecule .

number of TRAP-positive multinucleated cells was observed in group $\mathrm{Z}$ from 7 to 28 days postoperative. However, group $\mathrm{Z}$ presented a higher proportion of nonattached osteoclasts when compared with group $C$ at 7 as well at 28 days postoperative. Many of them were giant and hypernucleated osteoclasts. These results are corroborated by the findings of others studies (Kuroshima et al., 2012; Bi et al., 2010; Yang et al., 2005; Weinstein et al., 2009). Similar findings were observed in another study by Kuroshima et al. (2012) in which mice received either ZA or saline (control group) for 13 months. ZA therapy significantly increased the number of nonattached osteoclasts compared with the animals in the control group. Interestingly, in the contralateral intact side of the maxilla, nonattached osteoclasts were not detected in the control group, but more than half of the samples from the $\mathrm{Z}$ group had nonattached osteoclasts.

It has been demonstrated that BP-induced inhibition of bone resorption in vitro does not require apoptosis of osteoclasts (HalasyNagy et al., 2001). Therefore, the findings of our study and those from other animal (Yang et al., 2005; Bi et al., 2010; Kuroshima et al., 2012) and in vitro (Halasy-Nagy et al., 2001) studies support the statement by Weinsten et al. (2009) suggesting that nitrogen-containing BPs may inhibit bone resorption without decreasing the number of osteoclasts, and may even increase their number in cancellous bone. This is also consistent with several other animal and clinical studies, which have found that the number of osteoclasts is either maintained or increased with BP treatment (Balena et al., 1993; Eriksen et al., 2002).

Weinsten et al. (2009) also theorized that nitrogen-containing BP therapy prolongs osteoclast lifespan. According to Yang et al. (2005) increased osteoclast numbers could result if BP-treated osteoclasts, although poorly functional, persist longer than normal osteoclasts. The released calcium is an important signal for osteoclast death (Nielsen et al., 2007). According to Weinsten et al. (2009) inhibition of bone resorption by nitrogen-containing BPs would effectively diminish the signal for osteoclast death that results from calcium release during resorption, thereby prolonging their lifespan and allowing time for fusion with additional mononuclear progenitors.

It is important to emphasize that the significant increase in the number of TRAP-positive multinucleated cells in group $\mathrm{Z}$ compared 
with group C at 28 days postoperative cannot be assumed to correlate directly with an increase in the bone resorption process because more than half of those cells were nonattached to the bone surface. Osteoclasts must effectively adhere to the bone surface in order to resorb bone, so the nonattached osteoclasts observed in our study were probably not functional as 'bone eaters'. We therefore hypothesize that long-term therapy with nitrogencontaining BPs, such as ZA, increases the number of osteoclasts and promotes the formation of nonattached osteoclasts, especially giant, hypernucleated ones. These nonattached osteoclasts are not able to degrade bone but they may be important in performing other functions in bone remodeling. Increasing evidence points to a more complex identity for osteoclasts beyond their role as 'bone eaters'. Osteoclasts may directly regulate osteoblast differentiation, the hematopoietic stem cells niche, T cell activation, and skewing (Charles and Aliprantis, 2014). Much remains to be learned before the role of osteoclasts in all aspects of skeletal biology can be fully appreciated (Charles and Aliprantis, 2014). The results of our study reinforce the need to further investigate the influence of long-term therapy with nitrogen-containing BPs on the complex roles of osteoclasts. Knowledge of the molecular mechanisms involved in bone remodeling may provide a novel approach to the control and prevention of BRONJ.

\section{Conclusion}

Long-term therapy with IV ZA stimulated nonattached osteoclast formation in healthy extraction sockets in rats, thus decreasing local bone resorption. However, it did not influence bone formation by osteoblasts.

\section{Acknowledgements}

The authors thank Dr Tetuo Okamoto (in memoriam) for his invaluable contributions to this study. This research was supported by the "National Council of Technological and Scientific Development' - CNPq (485293/2007-2). The ZA was provided by NOVARTIS Biociências S.A., São Paulo, SP, Brazil.

\section{References}

Aghaloo TL, Kang B, Sung EC, Shoff M, Ronconi M, Gotcher JE, et al: Periodontal disease and bisphosphonates induce osteonecrosis of the jaws in the rat. J Bone Miner Res 26: e1871-e1882. http://dx.doi.org/10.1002/jbmr.379, 2011

Aguirre JI, Akhter MP, Kimmel DB, Pingel JE, Williams A, Jorgensen M, et al: Oncologic doses of zoledronic acid induce osteonecrosis of the jaw-like lesions in rice rats (Oryzomys palustris) with periodontitis. J Bone Miner Res 27(10): 2130-2143. http://dx.doi.org/10.1002/jbmr.1669, 2012

Ali-Erdem M, Burak-Cankaya A, Cemil-Isler S, Demircan S, Soluk M, Kasapoglu C, et al: Extraction socket healing in rats treated with bisphosphonate: animal model for bisphosphonate related osteonecrosis of jaws in multiple myeloma patients. Med Oral Patol Oral Cir Bucal 16(7): e879-e883, 2011

Balena R, Toolan BC, Shea M, Markatos A, Myers ER, Lee SC, et al: The effects of 2year treatment with the aminobisphosphonate alendronate on bone metabolism, bone histomorphometry, and bone strength in ovariectomized nonhuman primates. J Clin Investig 92(6): 2577-2586, 1993

Barasch A, Cunha-Cruz J, Curro FA, Hujoel P, Sung AH, Vena D, et al: Risk factors for osteonecrosis of the jaws: a case-control study from the CONDOR dental PBRN. J Dent Res 90(4): 439-444. http://dx.doi.org/10.1177/0022034510397196, 2011

Barba-Recreo P, Del Castillo Pardo de Vera JL, García-Arranz M, Yébenes L, Burgueño M: Zoledronic acid-related osteonecrosis of the jaws. Experimental model with dental extractions in rats. J Craniomaxillofac Surg 42(6): 744-750. http://dx.doi.org/10.1016/j.jcms.2013.11.005, 2014

Baron R, Ferrari S, Russell RG: Denosumab and bisphosphonates: different mechanisms of action and effects. Bone 48: e677-e692. http://dx.doi.org/10.1016/ j.bone.2010.11.020, 2011

Bi Y, Gao Y, Ehirchiou D, Cao C, Kikuiri T, Le A, et al: Bisphosphonates cause osteonecrosis of the jaw-like disease in mice. Am J Pathol 177(1): 280-290. http://dx.doi.org/10.2353/ajpath.2010.090592, 2010

Cardemil C, Thomsen P, Larsson Wexell C: Jaw bone samples from bisphosphonatetreated patients: a pilot cohort study. Clin Implant Dent Relat Res 17(2): e679-e691. http://dx.doi.org/10.1111/cid.12307, 2015
Charles JF, Aliprantis AO: Osteoclasts: more than 'bone eaters'. Trends Mol Med 20(8): 449-459. http://dx.doi.org/10.1016/j.molmed.2014.06.001, 2014

Diniz-Freitas M, López-Cedrún JL, Fernández-Sanromán J, García-García A, Fernández-Feijoo J, Diz-Dios P: Oral bisphosphonate-related osteonecrosis of the jaws: clinical characteristics of a series of 20 cases in Spain. Med Oral Patol Oral Cir Bucal 7(5): 751-758, 2012

Eriksen EF, Melsen F, Sod E, Barton I, Chines A: Effects of long-term risedronate on bone quality and bone turnover in women with postmenopausal osteoporosis. Bone 31(5): 620-625, 2002

Ferlito S, Puzzo S, Liardo C: Preventive protocol for tooth extractions in patients treated with zoledronate: a case series. J Oral Maxillofac Surg 69(6): e1-e4. http://dx.doi.org/10.1016/j.joms.2010.10.055, 2011

Gong X, Yu W, Zhao H, Su J, Sheng Q: Skeletal site-specific effects of zoledronate on in vivo bone remodeling and in vitro BMSCs osteogenic activity. Sci Rep 31(7): 36129. http://dx.doi.org/10.1038/srep36129, 2017

Halasy-Nagy JM, Rodan GA, Reszka AA: Inhibition of bone resorption by alendronate and risedronate does not require osteoclast apoptosis. Bone 29(6): 553-559, 2001

Hokugo A, Christensen R, Chung EM, Sung EC, Felsenfeld AL, Sayre JW, et al: Increased prevalence of bisphosphonate-related osteonecrosis of the jaw with vitamin D deficiency in rats. J Bone Miner Res 25(6): 1337-1349. http:// dx.doi.org/10.1002/jbmr.23, 2010

Howie RN, Borke JL, Kurago Z, Daoudi A, Cray J, Zakhary IE, et al: A model for osteonecrosis of the jaw with zoledronate treatment following repeated major trauma. PLoS One 10(7):e0132520. http://dx.doi.org/10.1371/journal.pone.0132520, 2015

Khan AA, Morrison A, Hanley DA, Felsenberg D, McCauley LK, O'Ryan F, et al: Diagnosis and management of osteonecrosis of the jaw: a systematic review and international consensus. J Bone Miner Res 30(1): 3-23. http://dx.doi.org/ 10.1002/jbmr.2405, 2015

Kim JW, Cha IH, Kim SJ, Kim MR: Biomarkers for bisphosphonate-related osteonecrosis of the jaw. Clin Implant Dent Relat Res 18(2): 281-291. http:// dx.doi.org/10.1111/cid.12297, 2016

Kobayashi Y, Hiraga T, Ueda A, Wang L, Matsumoto-Nakano M, Hata K, et al: Zoledronic acid delays wound healing of the tooth extraction socket, inhibits oral epithelial cell migration, and promotes proliferation and adhesion to hydroxyapatite of oral bacteria, without causing osteonecrosis of the jaw, in mice. J Bone Miner Metab 28(2): 165-175. http://dx.doi.org/10.1007/s00774-0090128-9, 2010

Kuroshima S, Go VA, Yamashita J: Increased numbers of nonattached osteoclasts after long-term zoledronic acid therapy in mice. Endocrinology 153(1): 17-28. http://dx.doi.org/10.1210/en.2011-1439, 2012

Kuroshima S, Yamashita J: Chemotherapeutic and antiresorptive combination therapy suppressed lymphangiogenesis and induced osteonecrosis of the jawlike lesions in mice. Bone 56(1): 101-109. http://dx.doi.org/10.1016/ j.bone.2013.05.013, 2013

Mackie PS, Fisher JL, Zhou H, Choong PF: Bisphosphonates regulate cell growth and gene expression in the UMR 106-01 clonal rat osteosarcoma cell line. $\mathrm{Br}$ J Cancer 84(7): 951-958. http://dx.doi.org/10.1054/bjoc.2000.1679, 2001

Mada EY, Santos AC, Fonseca AC, Biguetti CC, Neves FT, Saraiva PP, et al: Effects of green tea and bisphosphonate association on dental socket repair of rats. Arch Oral Biol 75: 1-7. http://dx.doi.org/10.1016/j.archoralbio.2016.12.001, 2017

Manrique N1, Pereira CC, Luvizuto ER, Sánchez Mdel P, Okamoto T, Okamoto R, et al: Hypertension modifies OPG, RANK, and RANKL expression during the dental socket bone healing process in spontaneously hypertensive rats. Clin Oral Investig 19(6): 1319-1327. http://dx.doi.org/10.1007/s00784-014-1369-0, 2015

Marino KL, Zakhary I, Abdelsayed RA, Carter JA, O'Neill JC, Khashaba RM, et al: Development of a rat model of bisphosphonate-related osteonecrosis of the jaw (BRONJ). J Oral Implantol 38: 511-518. http://dx.doi.org/10.1563/AAID-JOI-D11-00057, 2012

Marx RE: Pamidronate (Aredia) and zoledronate (Zometa) induced avascular necrosis of the jaws: a growing epidemic. J Oral Maxillofac Surg 61(9): 1115-1117, 2003

Nagata M, Messora M, Okamoto R, Campos N, Pola N, Esper L, et al: Influence of the proportion of particulate autogenous bone graft/platelet-rich plasma on bone healing in critical-size defects: an immunohistochemical analysis in rat calvaria. Bone 45(2): 339-345. http://dx.doi.org/10.1016/j.bone.2009.04.246, 2009

Nakagawa T, Ohta K, Kubozono K, Ishida Y, Naruse T, Takechi M, et al: Zoledronate inhibits receptor activator of nuclear factor kappa-B ligand-induced osteoclas differentiation via suppression of expression of nuclear factor of activated T-cell c1 and carbonic anhydrase 2. Arch Oral Biol 60(4): 557-565. http://dx.doi.org/ 10.1016/j.archoralbio.2014.09.012, 2015

Nielsen RH, Karsdal MA, Sorensen MG, Dziegiel MH, Henriksen K: Dissolution of the inorganic phase of bone leading to release of calcium regulates osteoclast survival. Biochem Biophys Res Commun 360(4): 834-839, 2007

Pan B, Farrugia AN, To LB, Findlay DM, Green J, Lynch K, et al: The nitrogen-containing bisphosphonate, zoledronic acid, influences RANKL expression in human osteoblast-like cells by activating TNF-alpha converting enzyme (TACE). J Bone Miner Res 19: 147-154. http://dx.doi.org/10.1359/jbmr.2004.19.1.147, 2004

Reid IR, Cornish J: Epidemiology and pathogenesis of osteonecrosis of the jaw. Nat Rev Rheumatol 8: e90-e96. http://dx.doi.org/10.1038/nrrheum.2011, 2011

Shalhoub V, Faust J, Boyle WJ, Dunstan CR, Kelley M, Kaufman S, et al: Osteoprotegerin and osteoprotegerin ligand effects on osteoclast formation from human peripheral blood mononuclear cell precursors. J Cell Biochem 72(2): 251-261, 1999 
Silva PG, Ferreira Junior AE, Teófilo CR, Barbosa MC, Lima Júnior RC, Sousa FB, et al: Effect of different doses of zoledronic acid in establishing of bisphosphonaterelated osteonecrosis. Arch Oral Biol 60(9): 1237-1245. http://dx.doi.org/ 10.1016/j.archoralbio.2015.05.015, 2015

Soundia A, Hadaya D, Esfandi N, Molon RS, Bezouglaia O, Dry SM, et al: Osteonecrosis of the jaws (ONJ) in mice after extraction of teeth with periradicular disease. Bone 90: 133-141. http://dx.doi.org/10.1016/j.bone.2016.06.011, 2016

Suda T, Takahashi N, Udagawa N, Jimi E, Gillespie MT, Martin TJ: Modulation of osteoclast differentiation and function by the new members of the tumor necrosis factor receptor and ligand families. Endocr Rev 20(3): 345-357. http:// dx.doi.org/10.1210/edrv.20.3.0367, 1999

Theodoro LH, Longo M, Ervolino E, Duque C, Ferro-Alves ML, Assem NZ, et al: Effect of low-level laser therapy as an adjuvant in the treatment of periodontitis induced in rats subjected to 5-fluorouracil chemotherapy. J Periodont Res 51(5): 669-680. http://dx.doi.org/10.1111/jre.12347, 2016
Viereck V, Emons G, Lauck V, Frosch KH, Blaschke S, Gründker C, et al: Bisphosphonates pamidronate and zoledronic acid stimulate osteoprotegerin production by primary human osteoblasts. Biochem Biophys Res Commun 291(3): 680-686. http://dx.doi.org/10.1006/bbrc.2002.6510, 2002

Weinstein RS, Roberson PK, Manolagas SC: Giant osteoclast formation and longterm oral bisphosphonate therapy. N Engl J Med 360(16): 53-62. http:|/ dx.doi.org/10.1056/NEJMoa0802633, 2009

Williams DW, Lee C, Kim T, Yagita H, Wu H, Park S, et al: Impaired bone resorption and woven bone formation are associated with development of osteonecrosis of the jaw-like lesions by bisphosphonate and anti-receptor activator of NF- $\kappa \mathrm{B}$ ligand antibody in mice. Am J Pathol 184(11): 3084-3093. http://dx.doi.org/ 10.1016/j.ajpath.2014.07.010, 2014

Yang Li C, Majeska RJ, Laudier DM, Mann R, Schaffler MB: High-dose risedronate treatment partially preserves cancellous bone mass and microarchitecture during long-term disuse. Bone 37(3): 287-295, 2005 